Research paper

\title{
Trajectories and air flow features of ski jump-generated jets
}

MICHAEL PFISTER (IAHR Member), Research and Teaching Associate, Laboratory of Hydraulic Constructions (LCH), Ecole

Polytechnique Fédérale de Lausanne (EPFL), CH-1015 Lausanne, Switzerland

Email: michael.pfister@epfl.ch (author for correspondence)

WILLI H. HAGER (IAHR Honorary Member), Professor, Laboratory of Hydraulics, Hydrology and Glaciology (VAW), ETH Zurich, CH-8092 Zurich, Switzerland

Email:hager@vaw.baug.ethz.ch

ROBERT M. BOES (IAHR Member), Director, Professor, Laboratory of Hydraulics, Hydrology and Glaciology (VAW), ETH Zurich, CH-8092 Zurich, Switzerland

Email:boes@vaw.baug.ethz.ch

\begin{abstract}
Ski jumps are frequently applied as spillways of high dams. The resulting jet impact location on the plunge pool surface is often distant from the dam toe so that the latter is protected from scouring. Furthermore, the jet disintegrates and disperses prior to its impact, thereby reducing the specific energy addition to the plunge pool. The present research addresses four aspects, based on three physical modelling campaigns: (1) geometry of upper and lower jet trajectories; (2) virtual jet take-off angles for the trajectory computations; (3) average and minimum cross-sectional air concentrations along the jet; and (4) general jet air concentration profiles. It is shown that the trajectory parabola may also be applied for negative jet take-off angles, and that these are smaller than the bucket angle. As for the air concentration distribution along the jet, tests indicate that the latter depends exclusively on the relative jet black-water core length.
\end{abstract}

Keywords: Air concentration; flip bucket; jet; ski jump; trajectory

\section{Introduction}

Ski jumps (consisting of a chute and a flip bucket) are frequently provided at the end of high-head spillways or bottom outlets (Fig. 1) to enhance energy dissipation and to avoid critical phenomena related to high-speed flow (Rajaratnam 1976, Vischer and Hager 1995, 1998, Khatsuria 2005, Novak et al. 2007). For particular applications, they may also include a horizontal deviation or insets at the take-off lip to deflect the jet or to improve its disintegration process. This leads to a spread of the jet decreasing its density due to air entrainment. The resulting larger jet-footprint on the plunge pool surface as well as the decreased density reduces the scour potential on a loose river bed (Canepa and Hager 2003, Pagliara et al. 2006). Such a loose bed is however rapidly washed out during operation. As for the remaining bare rock, disintegrated jets may amplify rock scour due to air entrainment into the plunge pool (Bollaert and Schleiss 2003a, 2003b). Although ski jumps were incorporated in many hydraulic schemes in the past, limited research on their basic air-water two-phase flow features is available, whereas the jet trajectories are well described.

Recent works on ski jumps include those of Juon and Hager (2000) with a literature review on past studies, and a preliminary physical model investigation on the plane and spatial features of ski jump jets. Heller et al. (2005) considered the two-dimensional ski jump trajectories, again based on physical modelling. The pressure distribution on the bucket, the virtual take-off angles of the lower and the upper trajectories, the trajectories themselves, and the choking limits of the bucket were derived. Scale effects were also considered; a correct modelling of the jet trajectories requires a minimum model approach flow depth of around $0.04 \mathrm{~m}$. Another issue regarding the applicability of model observations on prototype jets was given by Wahl et al. (2008), stating that prototype jet trajectories frequently differ from the often applied trajectory parabola as given by USBR (1987). In a debate of the latter publication, several Discussers including Heller and

Received 18 February 2013; accepted 10 December 2013/Open for discussion until 31 December 2014. 

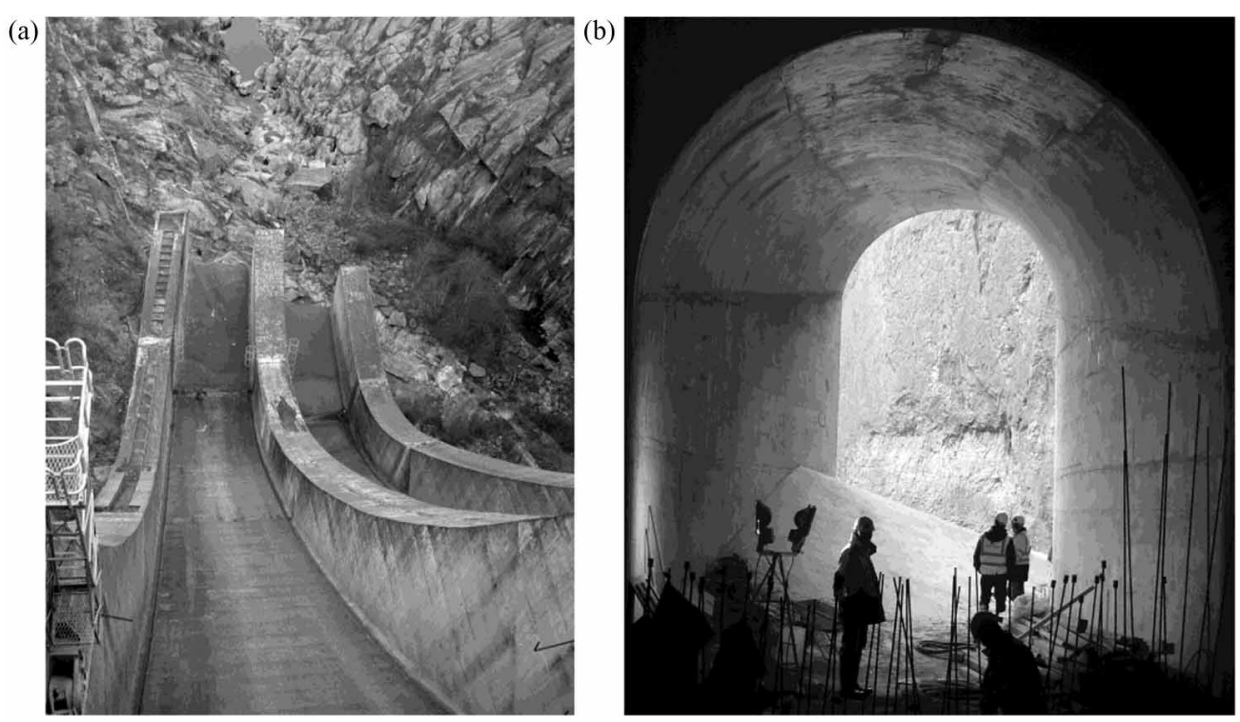

Figure 1 Ski jumps at (a) Venda Nova Dam spillway (Portugal) and (b) Kárahnjúkar Dam bottom outlet during construction (Iceland). Source: Photographs by M. Pfister

Pfister (2009) pointed to the effect of the effective take-off angle, which differs from the geometrical angle, and to the turbulence effect.

A milestone work regarding the jet air features was published by Ervine and Falvey (1987). They relate the turbulence intensity to the jet disintegration properties, in particular near the free surface. Furthermore, they found that the break-up length of the inner jet core is relevant for the air features, which is linked to the initial jet thickness. The air transport characteristics of jets issued from horizontal overfalls (without ski jump) downstream of bottom outlets was described by Toombes and Chanson (2007). They proposed a general jet air concentration profile based on the Gauss error function including a turbulence diffusion equation for air bubbles. The related experimental data furthermore indicate that only a negligible momentum loss occurs between the jet and its surrounding air. Schmocker et al. (2008) presented a model study of plane ski jump jets resulting particularly in a description of the jet air features, including preliminary general air profiles. They further described the effect of pre-aerated approach flow on the jet. However, the results are limited to horizontal chute bottoms upstream of the ski jump, which are rare in prototypes, as even bottom outlets often include a small chute slope for drainage purposes.

Steiner et al. (2008) presented the results of a similar physical model study as did Schmocker et al. (2008), but considered deflectors as the jet-generating element. A comparison of the results between the ski jump jets and these downstream of deflectors indicated relatively small differences, except for the maximum dynamic pressure head, which is larger at deflectors. Nevertheless, for deflector angles of $25-35^{\circ}$ relative to the horizontal chute bottom, the deflector results in a smaller integral pressure force because of the sharp-peaked dynamic pressure distribution. Although the total deflection angle of the flow is relatively small for deflectors, Pfister and Hager (2009) continued experimentation with deflector-generated jets including a much larger parameter variation. They proposed a normalization of all air features with the jet black-water core length, allowing for a comprehensive air concentration description along the jet. Besides, they described the jet trajectories with the take-off point and its maximum elevation, to avoid the criticized projectile approach. Pfister and Hager (2012) demonstrated that pre-aeration of the approach flow upstream of a jet-generating deflector influences the jet air features, as the jet black-water core length is reduced.

The chute upstream of a ski jump has a bottom angle $\phi$ relative to the horizontal (Fig. 2), with typical prototype values between almost horizontal for bottom outlets and some $50^{\circ}$ on gravity dams (Fig. 1). The approach flow to the ski jump is described in terms of its black-water depth $h_{o}$ and the Froude number $\mathrm{F}_{o}=$ $V_{o} /\left(g h_{o}\right)^{1 / 2}$, both defined immediately upstream (subscript $o$ ) of the ski jump. Here, $V_{o}=$ average approach flow velocity and $g=$ gravity constant. The geometry of the ski jump is given by its radius $R$ and its total deflection angle $\beta$. The streamwise lip angle relative to the horizontal, similar to the geometrical takeoff angle, is thus $\alpha=\beta-\varphi$, which is typically limited to $30^{\circ}$ (USBR 1987). A geometrical parameter related to the ski jump is the equivalent deflector angle $\tan \delta=(1-\cos \beta) / \sin \beta$. The height of the ski jump is $t=R(1-\cos \beta)$, defined perpendicular to the chute bottom.

The present research analyses the trajectories and air concentration characteristics of a plane jet downstream of a ski jump, based on raw data of previous investigations conducted by Heller (2004), Schmocker (2006), and Balestra (2012). By combining these three data sets, increased application limits are provided, allowing for a suitable use of the herein derived results on prototype conditions. 


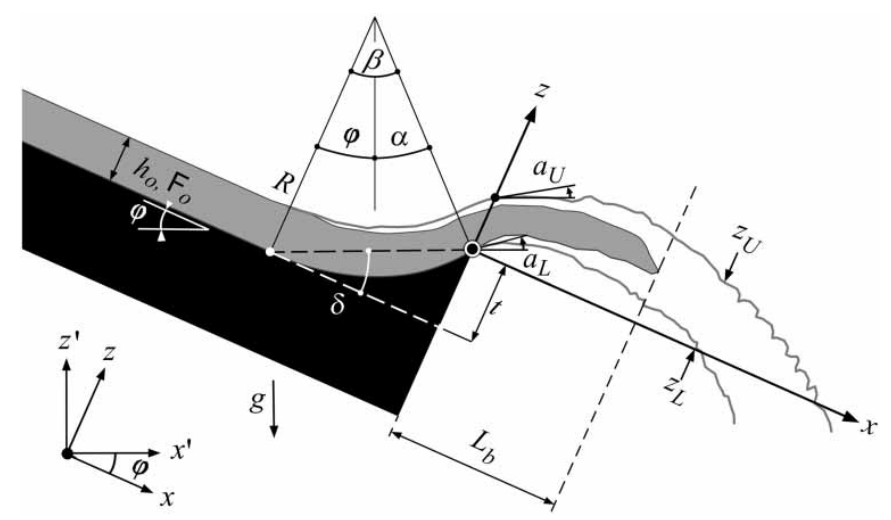

Figure 2 Definition sketch including relevant parameters of ski jump and jet

\section{Physical models}

\subsection{Physical models and test programme}

The data discussed herein were collected during three physical model investigations at the Laboratory of Hydraulics, Hydrology and Glaciology (VAW), ETH Zurich:

(1) The study of Heller (2004) was conducted in a horizontal $\left(\varphi=0^{\circ}\right)$ channel of $7 \mathrm{~m}$ length and $0.50 \mathrm{~m}$ width, later also used by Schmocker (2006) and Steiner et al. (2008). The discharge was supplied via a jet-box, allowing for an independent variation of discharge $Q$ and $h_{o}$. The approach flow was black-water, and the jet cavity was aerated to assure atmospheric pressure. Various ski jumps were installed some $2 \mathrm{~m}$ downstream of the jet-box, generating a plane-free jet.
Heller conducted 91 tests, including a wide spectrum of geometrical and hydraulic parameters, as listed by Heller et al. (2005). For the present analysis, only the trajectory data were considered, involving discharges of $Q \leq 185 \mathrm{l} / \mathrm{s}$.

(2) The study of Schmocker (2006) was conducted in the same channel as Heller (2004). The deflection angle of the ski jump was fixed at $\beta=30^{\circ}$ and its radius was $R=0.40 \mathrm{~m}$. The herein considered tests are listed in Table 1 (test numbers $\mathrm{O}$ to $\mathrm{S}$ ). Local air concentrations were measured, also to derive the jet trajectories (defined where the jet air concentration was $C=0.90$ ). Tests with pre-aeration of the approach flow were not considered herein, so that the average (subscript $a$ ) approach flow air concentration was $C_{a o} \approx 0.05$, with $Q \leq$ $1481 / \mathrm{s}$. For the air concentration data, the streamwise profile spacing was $0.08 \mathrm{~m} \leq \Delta x \leq 0.20 \mathrm{~m}$, with a spacing within the profiles of $\Delta z=5 \mathrm{~mm}$.

(3) The study of Balestra (2012) was conducted in a channel of $6 \mathrm{~m}$ length and $0.30 \mathrm{~m}$ width including a variable chute bottom angle $\varphi$, previously used by Pfister and Hager (2009, 2012). The ski jump was inserted $2 \mathrm{~m}$ downstream of the jetbox to achieve fully-developed flow, so that $4 \mathrm{~m}$ remained for jet flow. Variable ski jump geometries were tested, as shown in Table 1 (test numbers A to N). Local air concentrations were measured to derive the jet trajectories. Again, black-water approach flow was generated with $C_{a o} \approx 0.05$, with discharges of $Q \leq 1401 / \mathrm{s}$ supplied. The jet cavity below the jet was sufficiently aerated to assure atmospheric pressure. The inclined streamwise spacing for air concentration data was $0.05 \mathrm{~m} \leq \Delta x \leq 0.20 \mathrm{~m}$ and $20 \mathrm{~mm} \leq$ $\Delta z \leq 60 \mathrm{~mm}$.

Table 1 Combined test programmes of Balestra (2012) including tests A to N, and of Schmocker (2006) for tests $\mathrm{O}$ to $\mathrm{S}$

\begin{tabular}{|c|c|c|c|c|c|c|c|c|c|c|c|}
\hline $\begin{array}{l}\text { Test } \\
\text { no. }\end{array}$ & $\begin{array}{c}\varphi \\
\left(^{\circ}\right)\end{array}$ & $\begin{array}{c}R \\
(\mathrm{~m})\end{array}$ & $\begin{array}{c}\beta \\
\left(^{\circ}\right)\end{array}$ & $\begin{array}{c}\alpha \\
\left(^{\circ}\right)\end{array}$ & $\begin{array}{c}h_{o} \\
(\mathrm{~m})\end{array}$ & $\begin{array}{l}\mathrm{F}_{o} \\
(-)\end{array}$ & $\begin{array}{c}\delta \\
\left(^{\circ}\right)\end{array}$ & $\begin{array}{c}t \\
(\mathrm{~m})\end{array}$ & $\begin{array}{c}L_{b} \\
(\mathrm{~m})\end{array}$ & $\begin{array}{l}\mathrm{W}_{o} \\
(-)\end{array}$ & $\begin{array}{c}\mathrm{R}_{o} \\
\left(\times 10^{5}\right)\end{array}$ \\
\hline A & 12 & 0.400 & 12 & 0 & 0.050 & 8.01 & 6.0 & 0.009 & 0.381 & 146 & 2.50 \\
\hline (B) & 12 & 0.400 & 27 & 15 & 0.034 & 5.38 & 13.5 & 0.044 & 0.355 & 67 & 0.94 \\
\hline $\mathrm{C}$ & 12 & 0.400 & 27 & 15 & 0.044 & 6.06 & 13.5 & 0.044 & 0.323 & 97 & 1.56 \\
\hline D & 12 & 0.400 & 27 & 15 & 0.050 & 8.02 & 13.5 & 0.044 & 0.281 & 146 & 2.51 \\
\hline (E) & 12 & 0.400 & 42 & 30 & 0.037 & 4.91 & 21.0 & 0.103 & 0.139 & 66 & 0.96 \\
\hline $\mathrm{F}$ & 12 & 0.400 & 42 & 30 & 0.044 & 6.06 & 21.0 & 0.103 & 0.151 & 97 & 1.56 \\
\hline G & 12 & 0.200 & 27 & 15 & 0.044 & 6.06 & 13.5 & 0.022 & 0.307 & 97 & 1.56 \\
\hline $\mathrm{H}$ & 12 & 0.200 & 27 & 15 & 0.050 & 8.02 & 13.5 & 0.022 & 0.235 & 146 & 2.51 \\
\hline I & 30 & 0.400 & 12 & -18 & 0.038 & 8.06 & 6.0 & 0.009 & 0.363 & 112 & 1.67 \\
\hline $\mathrm{J}$ & 30 & 0.400 & 12 & -18 & 0.046 & 9.56 & 6.0 & 0.009 & 0.318 & 161 & 2.65 \\
\hline K & 30 & 0.400 & 27 & -3 & 0.037 & 8.03 & 13.5 & 0.044 & 0.203 & 127 & 1.88 \\
\hline $\mathrm{L}$ & 30 & 0.200 & 27 & -3 & 0.037 & 8.01 & 13.5 & 0.022 & 0.217 & 126 & 1.85 \\
\hline M & 30 & 0.200 & 27 & -3 & 0.046 & 9.55 & 13.5 & 0.022 & 0.218 & 161 & 2.65 \\
\hline $\mathrm{N}$ & 30 & 0.200 & 27 & -3 & 0.067 & 8.51 & 13.5 & 0.022 & 0.438 & 209 & 4.16 \\
\hline $\mathrm{O}$ & 0 & 0.400 & 30 & 30 & 0.045 & 5.00 & 15.0 & 0.054 & 0.432 & 82 & 1.34 \\
\hline (P) & 0 & 0.400 & 30 & 30 & 0.045 & 2.99 & 15.0 & 0.054 & 0.347 & 49 & 0.80 \\
\hline Q & 0 & 0.400 & 30 & 30 & 0.045 & 7.92 & 15.0 & 0.054 & 0.275 & 131 & 2.13 \\
\hline $\mathrm{R}$ & 0 & 0.400 & 30 & 30 & 0.070 & 5.10 & 15.0 & 0.054 & 0.985 & 130 & 2.64 \\
\hline (S) & 0 & 0.400 & 30 & 30 & 0.030 & 4.92 & 15.0 & 0.054 & 0.220 & 54 & 0.71 \\
\hline
\end{tabular}


In all three studies, the discharge $Q$ was measured using electromagnetic flow meters (Krohne, Germany), the flow depth $h_{o}$ using point gauges, and the local air concentrations $C$ with a fibre-optical probe (RBI, France) fixed on an automatic trolley with rails parallel to the chute bottom along the inclined coordinate $x$. The resulting air concentration measurements were also used to derive the upper (subscript $U$ ) and lower (subscript $L$ ) jet surfaces $z_{U}$ and $z_{L}$ defined along $C=0.90$, resulting in the jet trajectories. Table 1 details the test programme of Schmocker (2006) and Balestra (2012). Heller's (2004) tests are not listed for space reasons, and his tests with $h_{o}<0.04 \mathrm{~m}$ or $\mathrm{F}_{o}<3.8$ were ignored due to scale effects. The herein considered tests include thus the parameter ranges of $0^{\circ} \leq \varphi \leq 30^{\circ},-18^{\circ} \leq \alpha \leq$ $40^{\circ}, 10^{\circ} \leq \beta \leq 42^{\circ}, 0.09 \leq h_{o} / R \leq 0.95$, and $3.8 \leq \mathrm{F}_{o} \leq 10.1$.

\subsection{Scale effects}

Ervine and Falvey (1987) noted that aeration processes of free jets depend on the Weber $\mathrm{W}_{o}=\left(\rho V_{o}^{2} h_{o}\right) / \sigma$ and Reynolds $\mathrm{R}_{o}=$ $V_{o} h_{o} / v$ numbers. Here, $\rho=$ water density, $\sigma=$ water surface tension, and $v=$ water kinematic viscosity. The air transport characteristics of scale models are affected by scale effects because the internal flow turbulence, described by $\mathrm{R}_{o}$, is underestimated, whereas surface tension, given by $\mathrm{W}_{o}$, is overestimated (Chanson 2009). As a comprehensive dynamic similitude exists only at full-scale, the underestimation of the air transport is reduced if limitations of $\mathrm{W}_{o}$ or $\mathrm{R}_{o}$ are respected (Pfister and Chanson 2012). Thus, tests with $\mathrm{W}_{o}<80$ and $\mathrm{R}_{o}<1.3 \times 10^{5}$ were ignored herein (test numbers in parentheses in Table 1). Pfister and Hager (2012) discussed the similarity of primary and secondary jet disintegration, concluding that particularly the secondary disintegration is affected as the stable drop size seems constant. This effect mainly concerns the part beyond the $90 \%$ air concentrations, which is irrelevant to define the jet surface. Heller et al. (2005) identified scale effects regarding the jet trajectories for $h_{o} \leq 0.04 \mathrm{~m}$, whereas Juon and Hager (2000) report of $h_{o} \leq 0.05 \mathrm{~m}$.

\section{Trajectories}

\subsection{Trajectory geometry}

The studies of Schmocker (2006) and Balestra (2012) are considered, whereas that of Heller (2004) is ignored for the present trajectory computation. Given that Heller (2004) proposed the herein applied trajectory normalization, one can assume that his data also fit.

The trajectories are defined along the upper $z_{U}^{\prime}$ and the lower $z_{L}^{\prime}$ jet surfaces at $C=0.90$. All data were transformed to the horizontal $\left(x^{\prime} ; z^{\prime}\right)$ coordinate system by conducting a coordinate transformation. As proposed by Heller et al. (2005), the maximum (subscript $M$ ) jet elevations are considered for the trajectory normalization as $X^{\prime}=x^{\prime} / x_{M}^{\prime}$ and $Z^{\prime}=z^{\prime} / z_{M}^{\prime}$, with $x_{M}^{\prime}$ at $z_{M}^{\prime}$.
The maxima may be derived from the derivative of the trajectory parabola by setting $\mathrm{d} z^{\prime} / \mathrm{d} x^{\prime}=0$, resulting in the normalized trajectory coordinates

$$
\begin{aligned}
& X^{\prime}=\frac{2 x^{\prime}}{h_{o} \mathrm{~F}_{\mathrm{o}}^{2} \sin \left(2 \alpha_{j}\right)} \\
& Z^{\prime}=\frac{2 z^{\prime}}{h_{o} \mathrm{~F}_{\mathrm{o}}^{2}\left(\sin \alpha_{j}\right)^{2}}
\end{aligned}
$$

Here, $\alpha_{j}=$ virtual jet (subscript $j$ ) take-off angle used to fit the data to the general mass-point parabola. The latter results if the trajectory maxima at $X^{\prime}=Z^{\prime}=1$ and $X^{\prime}=Z^{\prime}=0$ as take-off location are respected, namely if

$$
Z^{\prime}=2 X^{\prime}-X^{\prime 2}
$$

The data from the physical models were thus all normalized with Eqs. (1) and (2), and then fitted to Eq. (3) by estimating the appropriate angle $\alpha_{j}$.

For positive values of $\alpha_{j}$, a jet maximum $z_{M}^{\prime}$ exists, so that the model data may - alternatively to Eq. (2) - be normalized with

$$
\begin{aligned}
& Z_{U}^{\prime}=\frac{z_{U}^{\prime}-h_{o}}{z_{M}^{\prime}-h_{o}} \\
& Z_{L}^{\prime}=\frac{z_{L}^{\prime}}{z_{M}^{\prime}}
\end{aligned}
$$

The data were fitted to Eq. (3) by varying $\alpha_{j}$. In Eq. (4), $h_{o}$ has to be subtracted from the measured upper trajectory, because the take-off location is otherwise at $\left(x^{\prime}=0 ; z^{\prime}=h_{o}\right)$. For the lower trajectory, the jet take-off is at the ski jump lip $\left(x^{\prime}=0 ; z^{\prime}=0\right)$, so that no correction is required (Eq. 5). The data of the upper and lower normalized trajectories and Eq. (3) are compared in Fig. 3a, resulting in a coefficient of determination of $R^{2}=0.94$. Note that Heller et al. (2005) reported $R^{2}=0.97$ for the upper and $R^{2}=0.96$ for the lower trajectories.

For negative values of $\alpha_{j}$, no jet maximum occurs. The model data $z^{\prime}$ of the upper and lower trajectories were then exclusively normalized with Eq. (2), either using a virtual upper or lower take-off angle $\alpha_{U}$ or $\alpha_{L}$ for $\alpha_{j}$. Note that Eq. (3) remains valid as the general trajectory function. The related data and Eq. (3) are compared in Fig. 3b, with $R^{2}=0.99$.

\subsection{Virtual take-off angle}

Various studies indicate that the effective jet take-off angle $\alpha_{\text {eff }}$ measured immediately downstream of the ski jump lip (expressed as $\left.\tan \alpha_{\text {eff }}=\left(\Delta z^{\prime} / \Delta x^{\prime}\right)\right)$ differs from the geometrical take-off angle $\alpha$ (e.g. Dhillon et al. 1981, Pfister 2012). Accordingly, using $\alpha$ for trajectory computation results in non-adequate, typically too long jets. In reality, the jet impact location on the plunge pool surface is closer at the dam than estimated with $\alpha$. To derive nevertheless reliable trajectories, particularly in the far-field of 
(a)

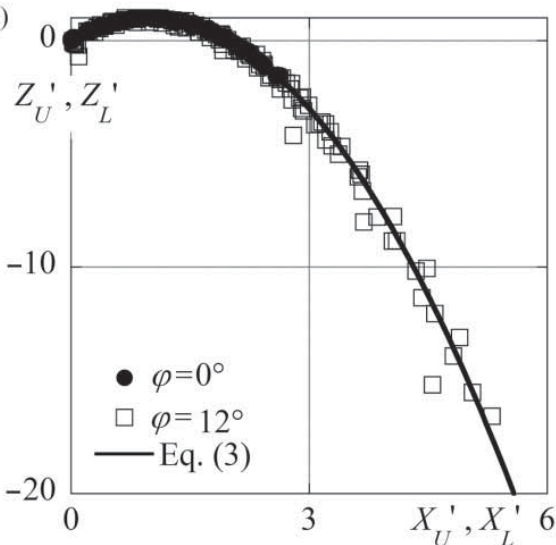

(b)

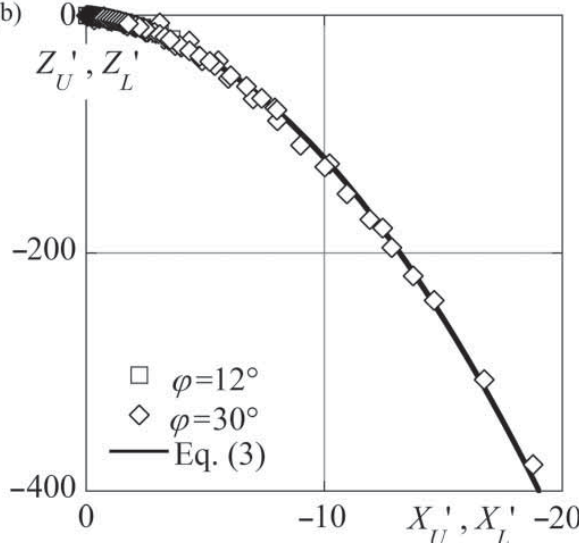

Figure 3 Normalized jet trajectories with (a) positive and (b) negative virtual jet take-off angles $\alpha_{j}$, both including data of Schmocker (2006) and Balestra (2012)
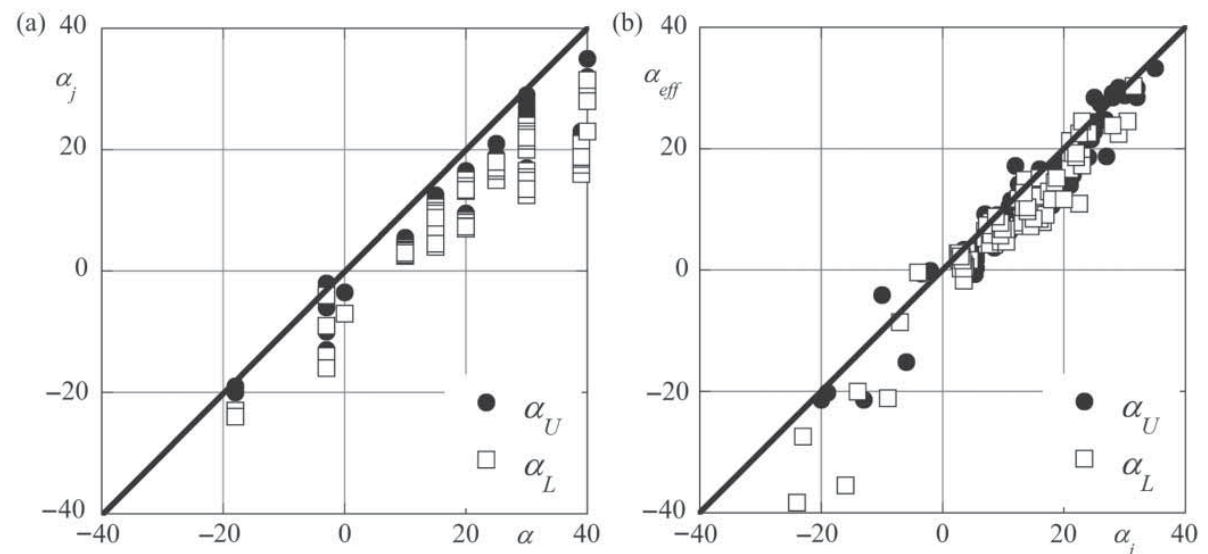

Figure 4 Comparison of (a) geometrical take-off angle $\alpha$ with virtual take-off angle $\alpha_{j}$, and (b) virtual take-off angle $\alpha_{j}$ with measured take-off angle $\alpha_{e f f}$

the jet, the approach of Heller et al. (2005) was selected herein, i.e. the trajectories were fitted to Eq. (2) by determining virtual $\alpha_{U}$ and $\alpha_{L}$. The latter are referred to as $\alpha_{j}$ in Eqs. (1) and (2). These virtual angles $\alpha_{j}$ are mostly significantly smaller than the geometrical values $\alpha$, as shown in Fig. 4a. The comparison of the virtual angles $\alpha_{j}$ with the effectively measured values $\alpha_{\text {eff }}$ indicates that these are similar but not identical $\left(R^{2}=0.92\right.$, Fig. $\left.4 \mathrm{~b}\right)$, particularly if considering positive values. Note again that $\alpha_{j}$ was derived in order to best fit the measured trajectories (and thus the jet-footprint location) and should be considered in Eqs. (1) and (2), instead of $\alpha$ or $\alpha_{\text {eff }}$.

For all three data sets, the general normalization parameter used to derive the virtual jet take-off angles follows as

$$
\Lambda=\tan \alpha\left(1-\frac{h_{o}}{R}\right)^{1 / 3} \quad \text { if }-0.32 \leq \Lambda \leq 0.84
$$

For the herein tested range of $0.09 \leq h_{o} / R \leq 0.95$ (including also the data of Heller 2004), the term $\left(1-h_{o} / R\right)^{1 / 3}$ varies between 0.97 and 0.37 . The virtual take-off angles $\alpha_{U}$ and $\alpha_{L}$, defined relative to the horizontal, are then given by linear functions of $\Lambda$ as

$$
\begin{aligned}
\tan \alpha_{U} & =0.84 \Lambda-0.04 \\
\tan \alpha_{L} & =0.80 \Lambda-0.07
\end{aligned}
$$

Note that the limits of Eqs. (7) and (8) are identical to those of Eq. (6). For Eq. (7) $R^{2}=0.92$, whereas $R^{2}=0.90$ for Eq. (8). For small $h_{o}$ and large $R$, the virtual jet take-off angle $\alpha_{j}$ is thus only marginally below the geometrical take-off angle $\alpha$, because the flow follows the bucket curvature. In contrast, large $h_{o}$ and small $R$ values generate small terms $\left(1-h_{o} / R\right)^{1 / 3}$, so that $\alpha_{j}$ is significantly smaller than $\alpha$. The latter predictions and the values used to generate reliable trajectories are compared in Fig. 5 for the upper and the lower virtual take-off angles.

A novelty of the herein presented approach is the inclusion of negative virtual take-off angles. Note further that for positive $\alpha_{j}$ values, the first jet portion with positive $Z^{\prime}$ was "flat" in the models for $\mathrm{F}_{o} \approx 4$, not following the parabola. Such conditions seem unfavourable and must be avoided; they are also close to choking flow conditions on the bucket (Heller et al. 2005). The 

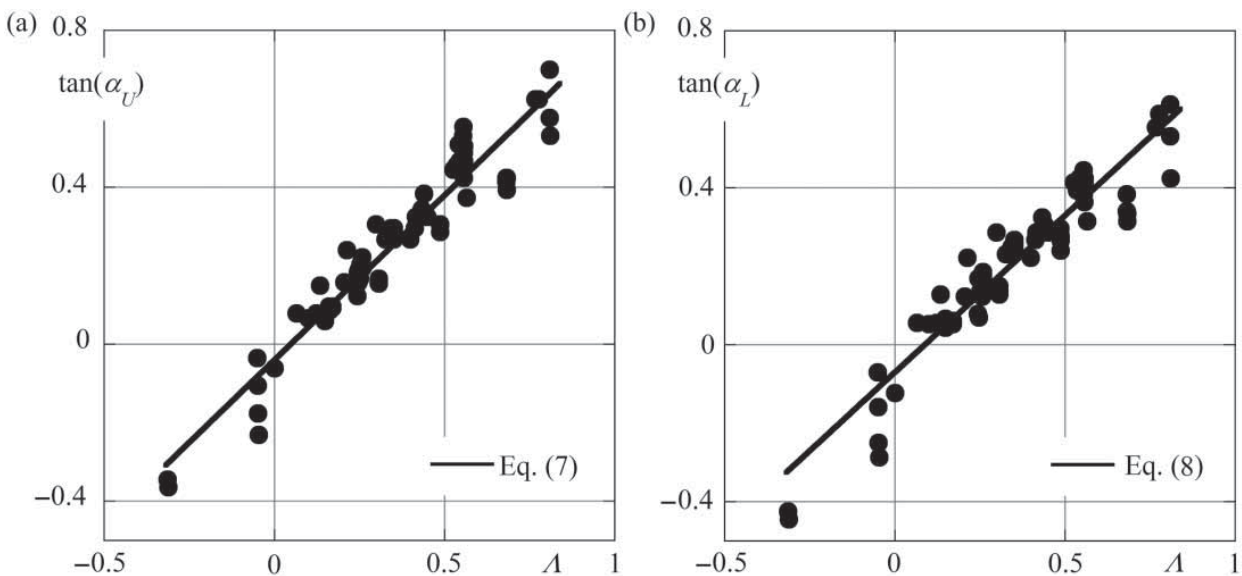

Figure 5 Virtual take-off angles as base of (a) upper, (b) lower jet trajectory computation, both including data of Heller (2004), Schmocker (2006), and Balestra (2012)

herein discussed virtual take-off angles are independent of chute slope $\varphi$, if the geometrical take-off angle $\alpha$ is defined between the horizontal and the take-off lip of the bucket (Fig. 2).

\section{Air features}

\subsection{Black-water core length}

Herein, only the studies of Schmocker (2006) and Balestra (2012) were considered, as Heller (2004) did not measure air concentrations. As demonstrated by Pfister and Hager (2009) for deflector-generated jets, their air concentration characteristics are described uniquely by the black-water (subscript $b$ ) core length $L_{b}$, an approach also selected herein. The latter is defined using the minimum (subscript $m$ ) air concentration $C_{m}$ measured within a certain air profile along the inclined coordinate $z$. A black-water core occurs between jet take-off at $x=0$ and the profile at location $x$ where $C_{m}=0.01$. Along $L_{b}$ values $C_{m}<0.01$ occur, whereas further downstream $C_{m}>0.01$. Note that $L_{b}$ was linearly interpolated between two neighbouring profiles with $C_{m}<0.01$ at the upstream profile and $C_{m}>0.01$ downstream.

Adapting the structure of the basic equation of Pfister and Hager (2009), i.e. their Eq. (12) giving the black-water core length of deflector-generated jets, generalizes the present data to

$$
\begin{aligned}
\frac{L_{b}}{h_{o}} & =76 \mathrm{~F}_{o}^{-1}(1+\tan \delta)^{-4}(1+\sin \varphi) \\
& =76 \Phi \text { if } 0.05 \leq \Phi \leq 0.13
\end{aligned}
$$

The modification of the basic equation relates to the inclusion of the deflector angle $\delta$ and an increase of its exponent from -0.5 to -4 . Ski jumps thus generate relatively shorter values of $L_{b}$ than equivalent deflectors, as the flow deviation is more pronounced. The effects of $F_{o}$ and $\varphi$ were found to be similar on both jet-generating structures. The data and Eq. (9) are compared in Fig. 6 , with $\Phi=\mathrm{F}_{o}^{-1}(1+\tan \delta)^{-4}(1+\sin \varphi)$ as abscissa. The

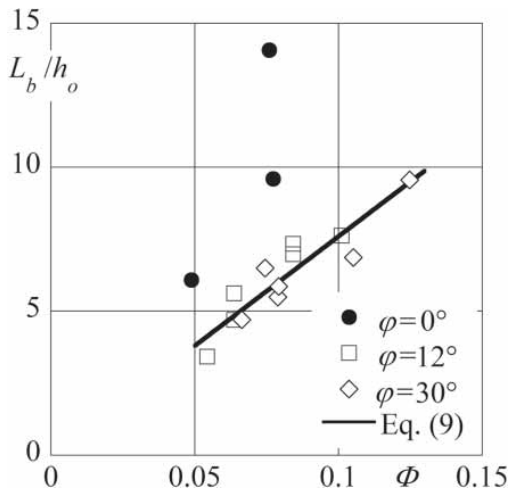

Figure 6 Relative black-water core length $L_{b} / h_{o}$ versus $\Phi$

data for $\varphi=12^{\circ}$ and $30^{\circ}$ collapse with Eq. (9) $\left(R^{2}=0.84\right)$, whereas those for $\varphi=0^{\circ}$ do not. This is a result of the decision to consider the basic equation of Pfister and Hager (2009), which was derived from an extensive data set including wide parameter ranges, instead of providing a new relationship including a smaller data base. In the further data analysis, exclusively the measured $L_{b}$ values are considered - and not the predictions according to Eq. (9) - so that the following remains valid also for $\varphi=0^{\circ}$.

With $L_{b}$ and the inclined coordinate $x$, the streamwise normalization for the air flow features is the relative black-water core length giving the location of a considered profile as multiple of $L_{b}$ as

$$
\chi=\frac{x}{L_{b}}
$$

Accordingly, $0<\chi<1$ corresponds to the black-water core portion, and $\chi \geq 1$ to the fully-aerated jet portion.

\subsection{Average and minimum concentrations}

Straub and Anderson (1958) defined the average (subscript $a$ ) air concentration $C_{a}$ as integral of the local values over the flow depth (per profile along the inclined coordinate $z$ ), herein equivalent to the jet thickness, with the boundaries at the upper $z_{U}$ and 
(a)

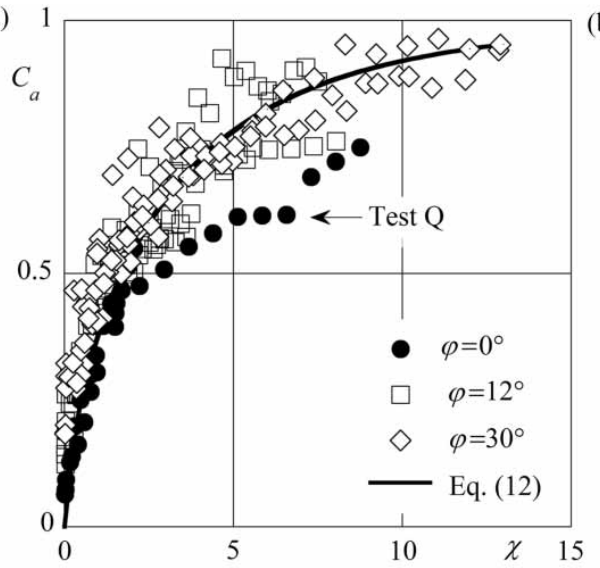

(b)

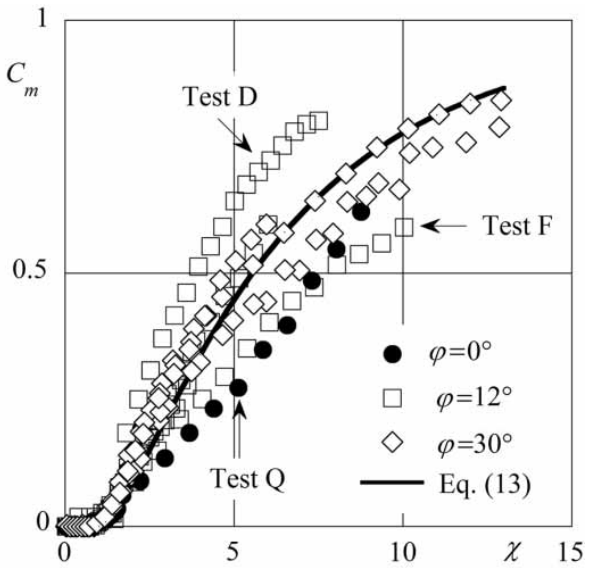

(c)

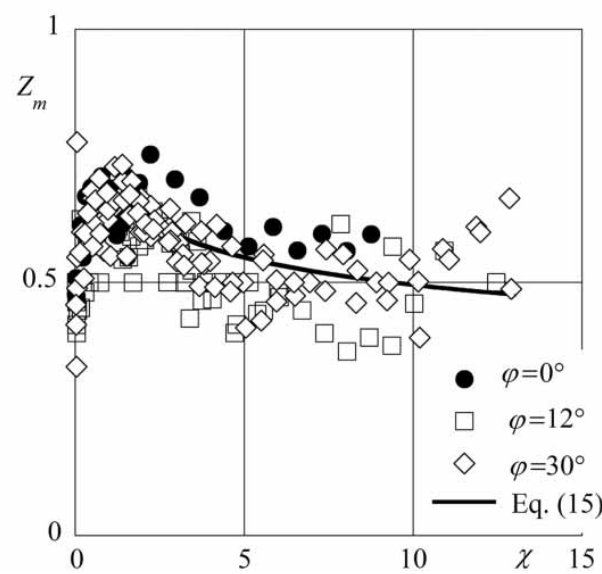

Figure 7 (a) Average air concentration $C_{a}(\chi)$, (b) minimum air concentration $C_{m}(\chi)$, (c) relative elevation $Z_{m}(\chi)$ of $C_{m}$

lower $z_{L}$ surface as

$$
C_{a}=\frac{1}{z_{U}-z_{L}} \int_{z_{L}}^{z_{U}} C(z) \mathrm{d} z
$$

The $C_{a}$ values versus the related individual $\chi$ values are shown in Fig. 7a; all data for all tests essentially collapse. Note that $C_{a}$ increases particularly along the first jet reach, and then tends to $C_{a} \rightarrow 1$. At $\chi=1.7$, for instance, $C_{a} \cong 0.50$; and $C_{a}=0.90$ at $\chi \cong 8$.8. The identical equation of Pfister and Hager (2009) may be applied to describe $C_{a}$ for ski jump jets, namely

$$
C_{a}=\tanh \left(0.4 \chi^{0.6}\right) \quad \text { if } 0 \leq \chi \leq 13
$$

Equation (12) yields $R^{2}=0.89$ with the values derived from the data by including all 221 recorded air profiles; whereas $R^{2}=0.91$ if Test No. Q is ignored, whose 13 data points are below Eq. (12).

The minimum air concentration $C_{m}$ within a profile is also exclusively a function of $\chi$. For non-aerated black-water approach flow, as tested herein, the initial values at $\chi=0$ correspond to $C_{m} \cong 0$ as observed at the chute bottom, and by definition it is $C_{m}=0.01$ at $\chi=1$. Further downstream, the $C_{m}$ values significantly increase to finally reach $C_{m} \rightarrow 1$ (Fig. 7b). In contrast to Pfister and Hager (2009), jets of $\chi>4$ are also considered herein. They follow, beside the pronounced increase along $1<\chi<5$, a much reduced inclination of the trend line for $\chi>10$, resulting in the generalized equation $\left(R^{2}=0.89\right)$

$$
C_{m}=\left[\tanh \left(0.4(\chi-1)^{0.6}\right)\right]^{2.5} \quad \text { if } 1 \leq \chi \leq 13
$$

Again, some tests indicate a reduced coefficient of determination, namely Tests No. D, F and Q (Fig. 7b), due to the sensitive determination of short $L_{b}$ values. If ignoring the latter tests, $R^{2}=$ 0.98 results.

To normalize the general air concentration profiles, the location $Z_{m}$ of $C_{m}$ within a profile is required. Setting the profile limits in the inclined $(x ; z)$ coordinate system at $z_{U}$ and $z_{L}$ results in

$$
Z=\frac{z-z_{L}}{z_{U}-z_{L}}
$$

so that $Z=1$ at the upper $\left(z_{U}\right)$ and $Z=0$ at the lower $\left(z_{L}\right)$ jet surfaces. Figure $7 \mathrm{c}$ shows $Z_{m}(\chi)$ with the ordinate as defined in Eq. (14). Note that the minima are located in the lower jet portion at jet take-off $(\chi=0)$, then at roughly $Z_{m}=0.7$ for $\chi \cong 1$, and finally tending to a symmetrical jet with $Z_{m}=0.5$ for $\chi>5$. The trend line for $Z_{m}$ is

$$
Z_{m}=0.7 \chi^{-0.15} \quad \text { if } 1 \leq \chi \leq 13
$$




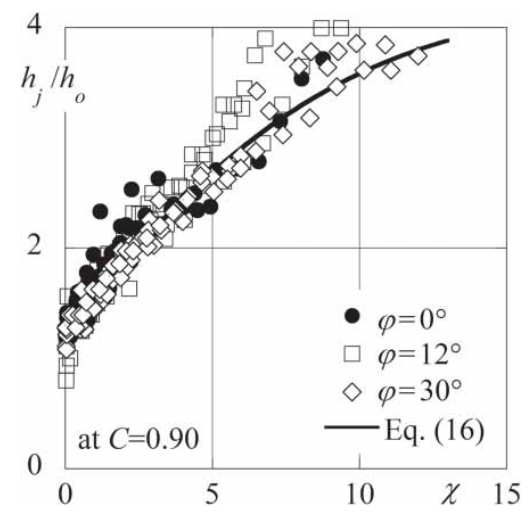

Figure 8 Relative jet thickness $h_{j} / h_{o}(\chi)$, based on $C=0.90$ concentration iso-lines as surfaces

The coefficient of determination is small due to the data scatter for large values of $\chi$, mainly because air profiles for $\chi>6$ are relatively "flat" so that an exact determination of $Z_{m}$ is difficult.

\subsection{Characteristic jet thickness}

The jet thickness $h_{j}=z_{U}-z_{L}$ (in the inclined coordinate system) was derived from a local subtraction of the lower from the upper jet surfaces. Herein, an alternative to the trajectory computation is given, because $C_{a}$ as a characteristic parameter for the jet spread also depends on $\chi$. In other words, the air transport along the jet is linked to its expansion in the flow direction, as noted by Ervine and Falvey (1987) for high-velocity jets.

The normalized jet thickness $h_{j} / h_{o}$ versus $\chi$ is shown in Fig. 8. At the jet take-off location, the observed flow depth is typically $h_{j} / h_{o} \cong 1.0$ to 1.3 , similar to the tested range of $1.02 \leq 1 / \cos \beta \leq 1.34$. Further downstream the jet spreads considerably. At a distance of $10 \chi$ its thickness is around $3.6 h_{o}$. The streamwise development of $h_{j} / h_{o}$ is given as $\left(R^{2}=0.99\right)$

$$
\frac{h_{j}}{h_{o}}=1.3+3 \tanh (0.1 \chi) \quad \text { if } 0 \leq \chi \leq 13
$$

Using the constant initial jet thickness $h_{j}=1.3 h_{o}$ at $\chi=0$ gives a similar approach of Eq. (16) as that with the geometrically corrected value $h_{j}=[1 / \cos \beta] h_{o}$, assuming a constant flow depth along the bucket.

\subsection{General concentration profiles}

So far, it was shown that the jet air features in terms of $C_{a}, C_{m}$ and $Z_{m}$ depend exclusively on $\chi$. It is thus evident that also the general air concentration profile $C(Z)$ varies exclusively with $\chi$, as shown in Fig. 9 at $\chi=1,2,4$, and 8. To include a reasonable amount of profiles, the aforementioned values of $\chi$ were selected in a range of $\pm 10 \%$. Note that the not shown data for $\chi=12$ are
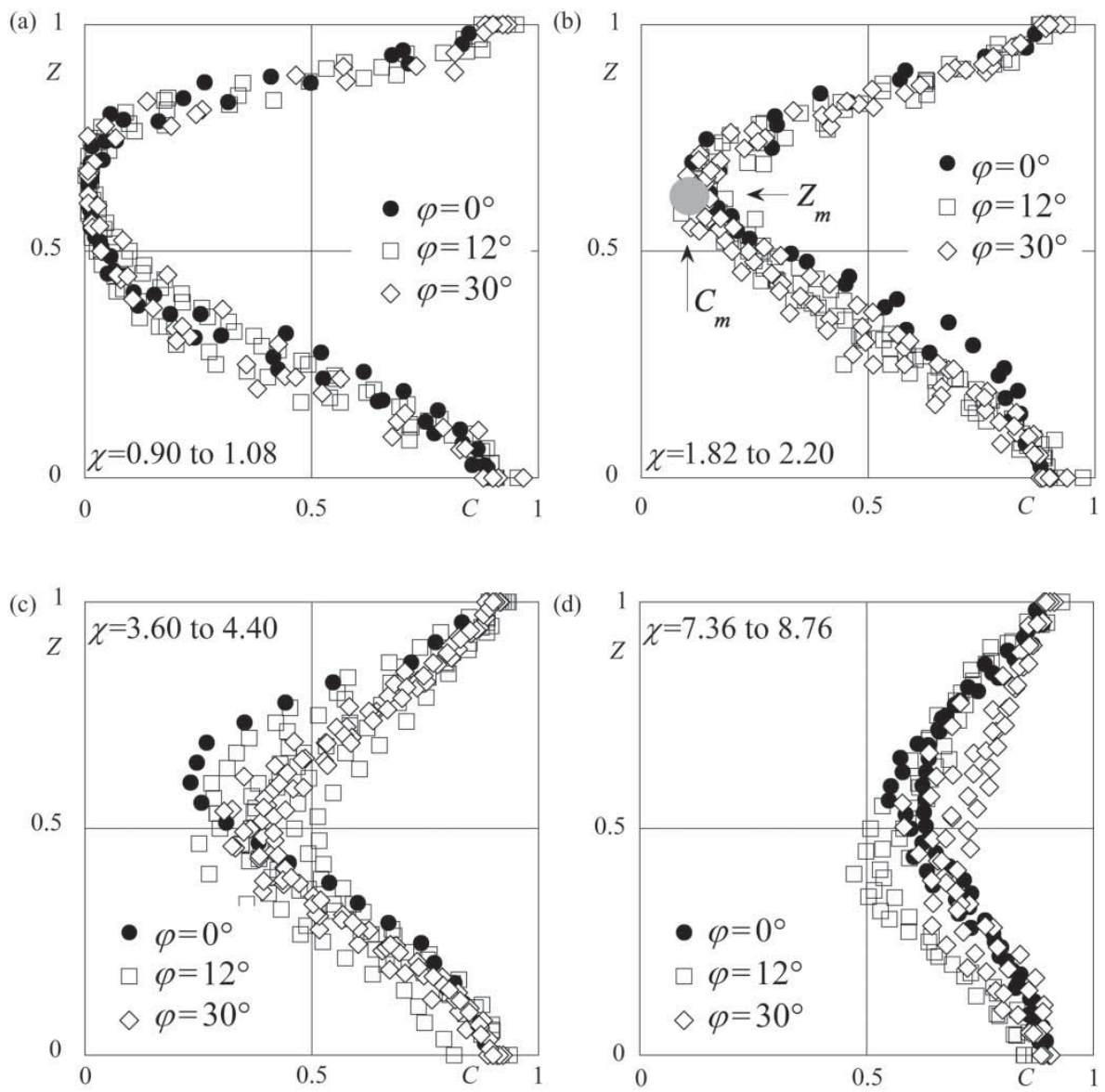

Figure 9 Cross-sectional jet air concentration profiles $C(Z)$ at $\chi=$ (a) 1 , (b) 2, (c) 4, and (d) 8 
similar to those at $\chi=8$. All data collapse for a certain value of $\chi$ even though the individual jet conditions vary significantly.

To derive a general function of the jet air concentration profile, the normalization process includes:

- At the jet surfaces, i.e. at $z_{U}$ and $z_{L}, C=0.90$. Thus, the upper fix-point is at $(Z=1 ; C=0.90)$, whereas the lower is $(Z=$ $0 ; C=0.90)$, and

- Within the jet, the values $\left(Z_{m} ; C_{m}\right)$ are considered as fix-points, as marked in Fig. 9b; these follow from Eqs. (13) and (15).

As for the abscissa of Fig. 9, i.e. regarding the normalization of $C$, the term

$$
\psi=\frac{C-C_{m}}{0.9-C_{m}}
$$

generates $\psi=1$ at the jet surfaces where $C=0.90$, and $\psi=0$ within the jet where $C=C_{m}$. As for the normalization of the ordinate, i.e. the location $Z$ of a certain value $C$, a distinction between the jet portion above $Z_{m}$ and that below has to be made. Locations in the upper jet portion between $Z=1$ and $Z_{m}$ are described by

$$
\zeta_{U}=\frac{Z-Z_{m}}{Z_{m}-1}+1 \quad \text { if } 1 \leq \chi \leq 13
$$

resulting in $\zeta_{U}=1$ at $Z_{m}$ and $\zeta_{U}=0$ at the upper jet surface at $Z=1$. For the lower jet portion, i.e. between $Z_{m}$ and the lower surface at $Z=0$, the normalization involves

$$
\zeta_{L}=\frac{Z}{Z_{m}} \quad \text { if } 1 \leq \chi \leq 13
$$

resulting in $\zeta_{L}=1$ at $Z_{m}$ and $\zeta_{L}=0$ at the lower jet surface defined at $Z=0$. Using this normalization, all $C(Z)$ data are included in Fig. 10, illustrating the general course of the air concentration profiles of the lower jet portion, and of the upper portion mirrored at the horizontal where $\zeta=1$. Again, the data essentially collapse, so that a trend function was fitted. Toombes

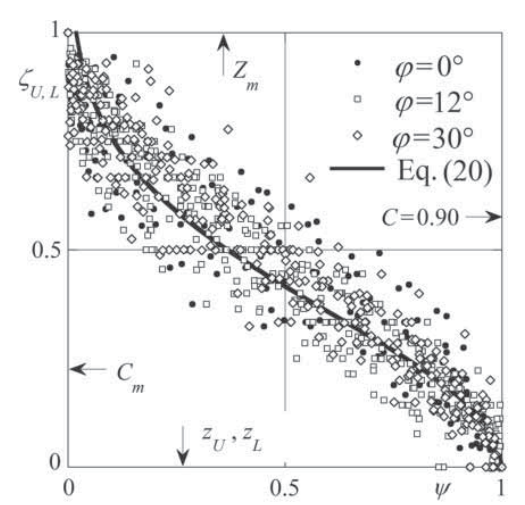

Figure 10 Normalized general air concentration profiles $\zeta_{U, L}(\psi)$ including both upper and lower jet portions and Chanson (2007) based their general air concentration profiles on the Gaussian error function $\pi^{0.5} \int \exp \left(-\zeta^{2}\right) \mathrm{d} \zeta$ including a diffusion term in $\zeta$. A slightly adapted equation was derived herein for all tested values $\chi$ as $\left(R^{2}=0.94\right)$

$$
\psi=\exp \left(-4 \zeta^{2}\right) \quad \text { if } 1 \leq \chi \leq 13
$$

\section{Discussion}

A certain difference between the model and the prototype trajectory is realistic, although tests with notable scale effects regarding air transport and trajectories were excluded herein. Heller et al. (2005) state: "In prototypes, the rising jet trajectories are supposed to follow essentially [herein Eq. (3)], whereas the falling trajectory portion deviates due to spray and wind." Pfister and Hager (2012) add: "If the jet is sufficiently long and issued with a large velocity, so that primary jet disintegration is fully established and the secondary becomes dominant, then a 'rupture' of the upper jet trajectory occurs." Primary jet disintegration is the disintegration of the jet itself under the influence of turbulence and gravity, whereas secondary disintegration describes the disintegration of water packages separated from the jet under aerodynamic interaction. Herein, both the jet length and the approach flow velocity are limited, so that neither rupture nor a significant jet deceleration was observed in the laboratory models. In addition, ski jumps are efficient for relatively high $\mathrm{F}_{o}$, which typically occur due to a considerable head usually combined with a long spillway. For the latter, aerators for cavitation prevention are installed or self-aeration of the flow occurs, so that the approach flow to the ski jump is pre-aerated. Then, the upper trajectory is typically lifted and the lower reduced (Pfister and Hager 2012), and the take-off velocity increased due to friction reduction (Minor 1987, Wood 1991). To summarize, a careful analysis of a prototype flow and a thorough engineering judgment are required when designing ski jumps.

The tests indicate that the chute bottom angle $\varphi$ has a priori no effect on the virtual take-off angle $\alpha_{j}$ as to consider for trajectory computation, if defining the geometrical take-off angle $\alpha$ relative to the horizontal (Fig. 2). The total jet dispersion angles $\alpha_{U}-\alpha_{L}$ at the jet take-off are between $1^{\circ}$ and $3^{\circ}$ for the tested ranges from Eqs. (7) and (8), for $-15^{\circ} \leq \alpha \leq 40^{\circ}$ and $0.1 \leq h_{o} / R \leq 0.9$. The angles are close to $3^{\circ}$ for large $\alpha$, with a minor effect of $h_{o} / R$. Accounting for the derivative of Eq. (16), then a total dispersion angle of $\tan \left[\left(h_{j} / h_{o}\right) \chi\right]=0.3$ results, or alternatively $\tan \left[\left(h_{j} / x\right)\left(L_{b} / h_{o}\right)\right]=0.3$. Considering the limitations of Eq. (9) gives total dispersion angles of approximately $1-2^{\circ}$. Thus, Eqs. (7) and (8) agree with Eq. (16), and with the value of $2.9^{\circ}$ noted by Schmocker et al. (2008) for non-pre-aerated jets. Heller et al. (2005) provide the jet dispersion as ratio $\alpha_{U} / \alpha_{L}=1.12$, whereas Eqs. (7) and (8) result in $1.09 \leq \alpha_{U} / \alpha_{L} \leq 1.56$ for $40^{\circ} \leq \alpha \leq 20^{\circ}$ and $0.1 \leq h_{o} / R \leq 0.9$, with a significant effect of $h_{o} / R$. The ratio $\alpha_{U} / \alpha_{L}$ is less reliable for small values $\alpha$, because then $\alpha_{L} \rightarrow 0$. 
The air concentration distribution within a jet is a function of its relative black-water core length $L_{b}$. The local jet air concentrations are high for short $L_{b}$, namely for: (1) large $\mathrm{F}_{o}$, linked to flow velocity and thus turbulence; (2) steep $\delta$, for a large deflection angle and a thus significant pressure relaxation downstream of jet take-off; and (3) flat $\varphi$, with a jet normal to gravity. The chute angle $\varphi$ thus affects the jet air flow features. This does not contradict the fact that the virtual take-off angle $\alpha_{j}$ is independent of $\varphi$, because $\alpha_{j}$ is a local value at the ski jump lip dominated by the relative curvature and $\alpha$, whereas $L_{b}$ is a parameter of the free jet under the influence of decreasing turbulence and gravity. Nevertheless, the concept of $L_{b}$ using the criterion $C_{m}=0.01$ is somehow arbitrary, as it applies only to relatively flat chutes (with bottom air concentrations below $C \cong 0.01$ ) and for non-pre-aerated approach flow.

The streamwise developments of $C_{a}$ and $C_{m}$ indicate that the: (1) increase of $C_{a}$ mainly takes place in the first jet reach, so that $C_{a}=0.50$ at $\chi=1.7$, whereas $C_{a}=0.75$ at $\chi=4.4$ or 2.6 times further downstream. The surface disintegration occurs thus primary in the first jet reach, where the turbulence from the upstream flow is still active; (2) jet core remains unaffected $\left(C_{m}<0.01\right)$ until the waves of both disintegrating surfaces merge (near $\chi=1$ ), typically at $0.7 \mathrm{Z}$. This is due to the chute flow turbulence and its amplification by the bucket, both primarily affecting the lower jet portion; (3) coherent jet core $\left(C_{m}<0.01\right)$ ends at $\chi \cong 1$ with $C_{a}=0.38$; (4) primary jet disintegration ends at $\chi \cong 5$, where the air profile is symmetrical $\left(C_{m}\right.$ at $\left.0.5 Z\right)$ and almost plain, with a marginal further increase of $C_{a}$.

The general air concentration profile across jets is similar to a bell-shaped curve, which is symmetrical for roughly $\chi>5$. Upstream of that limit, the upper and the lower jet portions include similar, but stretched profiles, both following an adapted Gaussian error function.

\section{Conclusions}

Ski jumps are a frequent spillway type, particularly for high heads and large discharges. They combine different advantages, whereas the effective trajectory geometry and the jet features at the impact onto the plunge pool surface are challenging to predict. The combined data of three physical model studies were thus analysed, focusing on the virtual jet take-off angle to derive reliable trajectories, and predicting the streamwise air flow features. The study particularly includes negative geometrical take-off angles of the bucket, to allow for a wider range of application.

The geometrical dimensions of a flip bucket are to be defined considering various conditions, among which the jet features are of primary concern. For practical reasons, a horizontal coordinate system was selected. The mass-point parabola approximates the effective trajectory well, but only if considering a virtual jet take-off angle smaller than the geometrical bucket angle. The virtual angle depends on the bucket geometry and on the approach flow depth, but not on the chute angle. It was shown that this concept even applies for negative virtual jet take-off angles. The jet dispersion angle is typically around $2-3^{\circ}$ if considering air concentrations of $90 \%$ as jet surfaces.

The air concentrations along the jet are uniquely linked to the relative black-water core length. This characteristic length describes the non-aerated inner jet extension and determines the average and minimum air concentrations within a jet profile. The jet black-water core length is influenced by the approach flow depth and Froude number, the equivalent deflector angle, and the chute bottom angle. The location of the minimum air concentration within a jet is first at the bottom near the takeoff lip as a result of the upstream chute flow, then migrates to the upper jet portion as turbulence immediately disintegrates the lower jet portion after take-off, and is finally at the jet centre due to the gravity effect. The global air concentration development suggests that the primary jet disintegration takes place in the very first jet portion mainly, particularly between the take-off and approximately five times the black-water core length. Further downstream, the air features hardly change anymore. A general air concentration profile across the jet is finally proposed, based on an equation similar to the Gaussian error function.

The results derived herein support the design process of ski jumps. Particularly the jet impact location onto the plunge pool surface may be derived, besides its air features at this point. The latter support the assessment of the plunge pool stability in terms of scour. The design of a ski jump includes additional criteria, as the maximum dynamic bottom pressures and bucket chocking.

\section{Acknowledgements}

Dr Valentin Heller, Imperial College London, is kindly acknowledged for having made available the raw data of his research to the authors. The authors further thank Dr Lukas Schmocker, Basler und Hofmann AG Zurich, and Mr Andrea A. Balestra, formerly ETH Zurich, now at Lombardi Engineering Minusio, for having provided their data sets.

\section{Notation}

$C=$ air concentration $(-)$

$\mathrm{F}_{o}=$ approach flow Froude number $(-)$

$g=$ gravity constant $\left(\mathrm{m} / \mathrm{s}^{2}\right)$

$h_{o}=$ approach flow depth (m)

$h_{j}=$ jet thickness (m)

$L_{b}=$ black-water core length (m)

$Q=$ discharge $\left(\mathrm{m}^{3} / \mathrm{s}\right)$

$R=$ bucket radius (m)

$\mathrm{R}_{o}=$ approach flow Reynolds number (-)

$t=$ bucket height $(\mathrm{m})$

$V_{o}=$ approach flow velocity $(\mathrm{m} / \mathrm{s})$

$\mathrm{W}_{o}=$ approach flow Weber number $(-)$

$x=$ inclined streamwise coordinate $(\mathrm{m})$

$x^{\prime}=$ horizontal streamwise coordinate $(\mathrm{m})$

$X^{\prime}=$ normalized horizontal streamwise coordinate $(-)$ 
$z=$ coordinate perpendicular to $x(\mathrm{~m})$

$z^{\prime}=$ vertical coordinate, perpendicular to $x^{\prime}(\mathrm{m})$

$Z=$ normalized jet thickness $(-)$

$Z^{\prime}=$ normalized vertical coordinate, perpendicular to $X^{\prime}(-)$

$\alpha=$ geometrical take-off angle relative to horizontal $\left({ }^{\circ}\right)$

$\alpha_{j}=$ virtual take-off angle relative to horizontal, either $\alpha_{U}$ or $\alpha_{L}\left(^{\circ}\right)$

$\beta=$ total bucket deflection angle $\left(^{\circ}\right)$

$\delta=$ equivalent deflector angle $\left(^{\circ}\right)$

$\zeta=$ normalization perpendicular to $\psi(-)$

$\Lambda=$ normalization for virtual take-off angle (-)

$v=$ water kinematic viscosity $\left(\mathrm{m}^{2} / \mathrm{s}\right)$

$\rho=$ water density $\left(\mathrm{kg} / \mathrm{m}^{3}\right)$

$\sigma=$ water surface tension $(\mathrm{N} / \mathrm{m})$

$\varphi=$ chute bottom angle relative to horizontal $\left(^{\circ}\right)$

$\Phi=$ normalization of black-water core length (-)

$\chi=$ relative black-water core length $(-)$

$\psi=$ normalization of air concentration $(-)$

$a=$ average

$b=$ black-water

$j=$ jet

$L=$ lower

$m=$ minimum

$M=$ maximum

$o=$ approach flow

$U=$ upper

\section{References}

Balestra, A.A. (2012). Einfluss von Zuflussgefälle auf Skisprünge [Effect of chute slope on ski jumps]. Master Thesis, VAW, ETH Zurich (unpublished, in German).

Bollaert, E.F.R., Schleiss, A.J. (2003a). Scour of rock due to the impact of plunging high velocity jets 1: A state-of-the-art review. J. Hydraulic Res. 41(5), 451-464.

Bollaert, E.F.R., Schleiss, A.J. (2003b). Scour of rock due to the impact of plunging high velocity jets 2: Experimental results of dynamic pressures at pool bottoms and in one- and two-dimensional closed end rock joints. J. Hydraulic Res. 41(5), 465-480.

Canepa, S., Hager, W.H. (2003). Effect of jet air content on plunge pool scour. J. Hydraulic Eng. 129(5), 358-365.

Chanson, H. (2009). Turbulent air-water flows in hydraulic structures: Dynamic similarity and scale effects. Environ. Fluid Mech. 9(2), 125-142.

Dhillon, G.S., Sakhuja, V.S., Paul, T.C. (1981). Measures to contain throw of flip bucket jet in installed structures. Irrigation and Power 38(3), 237-245.

Ervine, D.A., Falvey, H.T. (1987). Behavior of turbulent water jets in the atmosphere and in plunge pools. Proc. Inst. Civil Eng. 83(2), 295-314.

Heller, V. (2004). Einfluss des Absprungwinkels bei Skisprüngen des Wasserbaus [Effect of take-off angle at ski jumps in hydraulic engineering]. Master Thesis, VAW, ETH Zurich (unpublished, in German).
Heller, V., Pfister, M. (2009). Discussion of Computing the trajectories of free jets. J. Hydraulic Eng. 135(7), 622-623.

Heller, V., Hager, W.H., Minor, H.-E. (2005). Ski jump hydraulics. J. Hydraulic Eng. 131(5), 347-355.

Juon, R., Hager, W.H. (2000). Flip bucket without and with deflectors. J. Hydraulic Eng. 126(11), 837-845.

Khatsuria, R.M. (2005). Hydraulics of spillways and energy dissipators. Dekker, New York.

Minor, H.-E. (1987). Erfahrungen mit Schussrinnenbelüftung [Experiences with chute aerators]. Wasserwirtschaft 77(6), 292-295 (in German).

Novak, P., Moffat, A.I.B., Nalluri, C., Narayanan, R. (2007). Hydraulic structures. ed. 2. Taylor \& Francis, London.

Pagliara, S., Hager, W.H., Minor, H.-E. (2006). Hydraulics of plane plunge pool scour. J. Hydraulic Eng. 132(5), $450-461$.

Pfister, M. (2012). Jet impact angle on chute downstream of aerator. $4^{\text {th }} I A H R$ International Symposium on Hydraulic Structures, Porto, 7, 1-9 (CD-Rom).

Pfister, M., Chanson, H. (2012). Discussion of Scale effects in physical hydraulic engineering models. J. Hydraulic Res. 50(2), 244-246.

Pfister, M., Hager, W.H. (2009). Deflector-generated jets. J. Hydraulic Res. 47(4), 466-475.

Pfister, M., Hager, W.H. (2012). Deflector-jets affected by preaerated approach flow. J. Hydraulic Res. 50(2), 181-191.

Rajaratnam, N. (1976). Turbulent jets. Elsevier, New York.

Schmocker, L. (2006). Belüftungs-Eigenschaften von Skisprüngen [Aeration characteristics of ski jumps]. Master Thesis, VAW, ETH Zurich (unpublished, in German).

Schmocker, L., Pfister, M., Hager, W.H., Minor, H.-E. (2008). Aeration characteristics of ski jump jets. J. Hydraulic Eng. 134(1), 90-97.

Steiner, R., Heller, V., Hager, W.H., Minor, H.-E. (2008). Deflector ski jump hydraulics. J. Hydraulic Eng. 134(5), $562-571$.

Straub, L.G., Anderson, A.G. (1958). Experiments on selfaerated flow in open channels. J. Hydraulics Div. 84(7), $1-35$.

Toombes, L., Chanson, H. (2007). Free-surface aeration and momentum exchange at bottom outlet. J. Hydraulic Res. 45(1), $100-110$.

USBR (1987). Design of small dams. ed. 3. Bureau of Reclamation, US Department of the Interior, Denver.

Vischer, D.L., Hager, W.H. (1995). Energy dissipators. Balkema, Rotterdam.

Vischer, D.L., Hager, W.H. (1998). Dam hydraulics. Wiley, Chichester.

Wahl, T.L., Frizell, K.H., Cohen, E.A. (2008). Computing the trajectories of free jets. J. Hydraulic Eng. 134(2), 256-260.

Wood, I.R. (1991). Free surface air entrainment on spillways. In IAHR hydraulic structures design manual no. 4 (Air entrainment in free-surface flows), 55-84, I.R. Wood, ed. A.A. Balkema, Rotterdam. 